ARTICLE

DOI: $10.1038 /$ s41467-018-05162-z

\title{
Programmed loading and rapid purification of engineered bacterial microcompartment shells
}

\author{
Andrew Hagen ${ }^{1}$, Markus Sutter (D) ${ }^{1,2}$, Nancy $\operatorname{Sloan}^{1}$ \& Cheryl A. Kerfeld (10) 1,2,3
}

Bacterial microcompartments (BMCs) are selectively permeable proteinaceous organelles which encapsulate segments of metabolic pathways across bacterial phyla. They consist of an enzymatic core surrounded by a protein shell composed of multiple distinct proteins. Despite great potential in varied biotechnological applications, engineering efforts have been stymied by difficulties in their isolation and characterization and a dearth of robust methods for programming cores and shell permeability. We address these challenges by functionalizing shell proteins with affinity handles, enabling facile complementation-based affinity purification (CAP) and specific cargo docking sites for efficient encapsulation via covalent-linkage (EnCo). These shell functionalizations extend our knowledge of BMC architectural principles and enable the development of minimal shell systems of precisely defined structure and composition. The generalizability of CAP and EnCo will enable their application to functionally diverse microcompartment systems to facilitate both characterization of natural functions and the development of bespoke shells for selectively compartmentalizing proteins.

\footnotetext{
${ }^{1}$ Molecular Biophysics and Integrated Bioimaging Division, Lawrence Berkeley National Laboratory, 1 Cyclotron Road, Berkeley, CA 94720, USA. ${ }^{2}$ MSU-DOE Plant Research Laboratory, Michigan State University, 612 Wilson Road, East Lansing, MI 48824, USA. ${ }^{3}$ Department of Biochemistry and Molecular Biology, Michigan State University, 603 Wilson Road, East Lansing, MI 48824, USA. Correspondence and requests for materials should be addressed to C.A.K. (email: ckerfeld@lbl.gov)
} 
B acterial microcompartments (BMCs) are ensembles of enzymes enclosed in a semi-permeable proteinaceous shell, compositionally distinguishing them from the more familiar lipid-membrane bound organelles of eukaryotes. These compartments have been bioinformatically identified in the majority of bacterial phyla ${ }^{1}$ where they perform distinct, spatially separate metabolic functions allowing their hosts to fix carbon, as in the anabolic carboxysomes, or metabolize substrates like ethanolamine that generate volatile or toxic intermediates, as in the catabolic metabolosomes.

The shells of all characterized BMCs include a suite of cyclically symmetric protein building blocks which are hexagonal or pentagonal in shape and which together assemble into icosahedral bodies $^{2,3}$. The hexagons are hexa- or trimeric oligomers of a conserved, single or tandem-duplicated protein domain (pfam00936) that constitute the facets of the shell. Pentamers formed by the pfam03319 domain occupy the vertices. Pores in the hexamer and trimer (BMC-H and BMC-T) subunits mediate passage of small molecules through the shells ${ }^{4,5}$. In all characterized BMCs, the presence of multiple BMC-H and/or BMC-T homologs, each with distinct pore-lining residues, indicate differences in selectivity of transport among these subunits. In contrast to their roles in molecular selectivity, the structural necessity of multiple homologs remains underexplored. Pentamers are a minor component of shells as they occupy only the 12 vertex positions and are not thought to play a role in transport, yet are critical to the shell's function as a diffusive barrier ${ }^{6}$. The enzyme core of BMCs is assembled into the interior of shells through non-covalent interactions between short encapsulation peptides (EPs) on the cargo and the lumenal side of shell proteins $^{7-9}$. A second mode of encapsulation involves piggybacking of EP-less enzymes via interactions with EP-harboring proteins $^{10}$.

The genetic organization of BMC core and shell proteins into superloci ${ }^{1}$ and evidence of horizontal transfer among bacterial species $^{11,12}$ denote BMCs as genetically encoded metabolic modules. Genetic transplantation of such loci has been shown to confer novel metabolic functions to the recipient allowing, for example, Escherichia coli to grow on 1,2-propanediol after transfer of the $p d u$ operon from Citrobacter freundii ${ }^{13}$. Indeed, repurposing BMCs to encapsulate non-native pathways could allow metabolic engineers to improve titers by increasing enzyme stability, improving flux by concentrating intermediates and mitigating diversion of substrates to competing pathways, and sequestering potentially toxic or inhibitory intermediates ${ }^{14-16}$. Moreover, the nanoscale dimensions and greatly improved understanding of structure and assembly principles described recently ${ }^{2}$ suggest $\mathrm{BMC}$ shells are scaffolds fit for refactoring towards biomedical applications like polyvalent antigen display, drug delivery, and imaging-applications that have traditionally been the purview of other nanocompartments such as virus-like particles and ferritins ${ }^{17,18}$.

Hampering these ambitions are several technical challenges. Preparations of BMCs or BMC shells are laborious and typically result in low yields of heterogeneous particles (with some notable exceptions ${ }^{19,20}$ ). Targeting heterologous cargo to the lumen of shells using EPs that are derived from naturally occurring BMCs is inefficient, with western blotting often required to identify cargo in shell preparations ${ }^{19-22}$. EPs may also lead to aggregation, potentially compromising enzyme activity ${ }^{23}$, and the binding site of EPs is unknown so cargo loading in shells is not readily programmable. Furthermore, there is no disassembly/reassembly methods developed which would allow in vitro encapsulation of cargo.

Here, through rational modifications to shell proteins in our model BMC system HO (from Haliangium ochraceum), we report the development of the CAP and EnCo methods. Complementation-based Affinity Purification (CAP) enables facile screening of assembly and rapid purification of shells. We apply this method to demonstrate a remarkable plasticity of subunit composition in $\mathrm{HO}$ shells. Encapsulation via Covalentlinkage (EnCo) is used to robustly program multiple cargo species into shells at predictable ratios both in vivo and ex vivo, enabling the encapsulation of heterologously produced cargo and abiotic materials. Finally, we apply a fluorescence-based probe to assess the permeability of our engineered shells and find they maintain their barrier functionality against macromolecular species while permitting ingress of a small molecule-likely through the pores of one or more shell proteins. These methodological advances should be applicable to other BMC systems, simplifying and accelerating assembly and permeability studies as well as elevate BMC shells to the pantheon of well-characterized and engineerable nanoparticles, enabling their application towards metabolic engineering and biomedical applications.

\section{Results}

Shells lacking pentamers can be capped and affinity purified. To obtain a crystal structure of synthetic BMC shells it was necessary to supplement shells with additional copies of the pentamer-forming proteins. This suggests substoichiometric production of pentamer during expression of the Haliangium ochraceum (HO) shell synthetic operon ${ }^{2}$. Previously, we had observed that facets assembled into three-dimensional particles independent of the presence of pentamers ${ }^{20}$; taken together, these findings suggested a strategy for producing shells lacking vertices that could be subsequently capped and purified. We hypothesized that appending a high affinity, specific tag such as the Strep-II sequence $^{24}$ to the pentamer $\left(\mathrm{P}_{\mathrm{SII}}\right)$ would enable pull-downs of shells after capping. Additionally, affixing the ten residues, $1.2 \mathrm{kDa}$ Strep-II tag to the pentamer would result in a size shift allowing it to be resolved from the $10.1 \mathrm{kDa}$ hexamer protein via SDS-PAGE analysis. These combined properties would enable rapid purification and analysis of shells by obviating the need for laborious and technically demanding density gradient ultracentrifugation and ion-exchange chromatography steps.

We validated this hypothesis by comparing classically ${ }^{2,20}$ prepared $\mathrm{HO}$ shells comprised of a single hexamer and three trimer homologs $\left(\mathrm{HT}_{1} \mathrm{~T}_{2} \mathrm{~T}_{3}\right.$ shells; uncapped shells were used because the co-migrating pentamer and hexamer bands preclude fair comparison between samples) to capped $\mathrm{HT}_{1} \mathrm{~T}_{2} \mathrm{~T}_{3} \mathrm{P}_{\text {SII }}$ shells prepared by two methods. In the first method, which recapitulates normal in vivo assembly of shells, $\mathrm{P}_{\text {SII }}$ proteins were co-expressed from a secondary plasmid in the same strain, and then purified via a StrepTrap column. In the second method, ex vivo capping, clarified lysates of the $\mathrm{HT}_{1} \mathrm{~T}_{2} \mathrm{~T}_{3}$ expressing strain and a separate strain expressing $\mathrm{P}_{\mathrm{SII}}$ were mixed and after a brief incubation, purified via StrepTrap. Excess $\mathrm{P}_{\text {SII }}$ proteins and trace contaminants were subsequently removed via anion-exchange chromatography (see Methods for details and Supplementary Figs. 1-3 for complete purification schemes and tracking). Figure la shows that $\mathrm{HT}_{1} \mathrm{~T}_{2} \mathrm{~T}_{3} \mathrm{P}_{\mathrm{SII}}$ shells are obtained when either the in vivo (lane 2) or ex vivo (lane 3) capping/pull-down methods are employed according to SDS-PAGE analysis. We observe no morphological changes to our affinity-purified shells (Fig. 1b-d), indicating that the Strep-II-tag is well-tolerated. It is unclear why there are subtle differences in the abundance of contaminants between lanes 2 and 3 (e.g., the band migrating between 50 and $75 \mathrm{kDa}$ ), however these observations are reproducible and may arise from the different plasmid content of the strains used (a doubletransformed strain vs. a mixture of two singly-transformed strains). Nevertheless, the classically prepared shells are $\sim 70 \%$ 
a

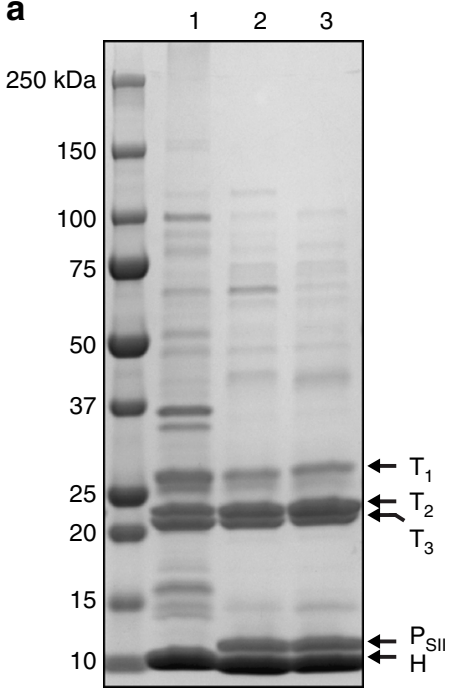

b

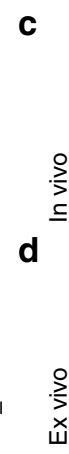

C
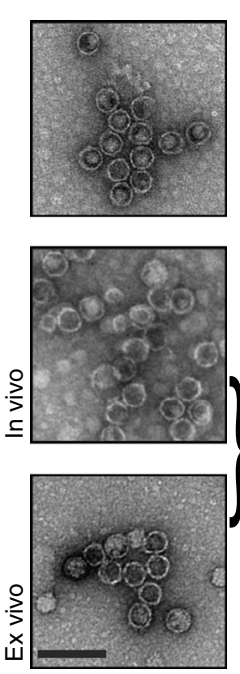
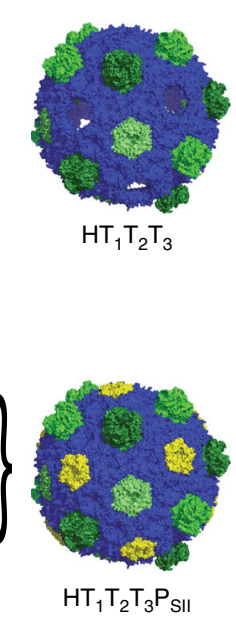

\section{f}

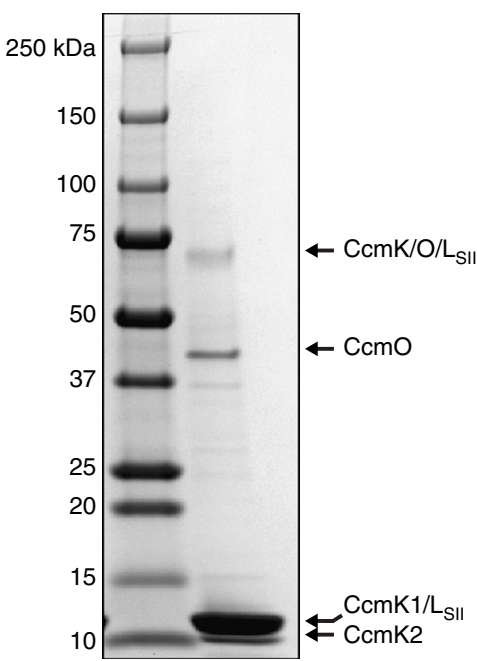

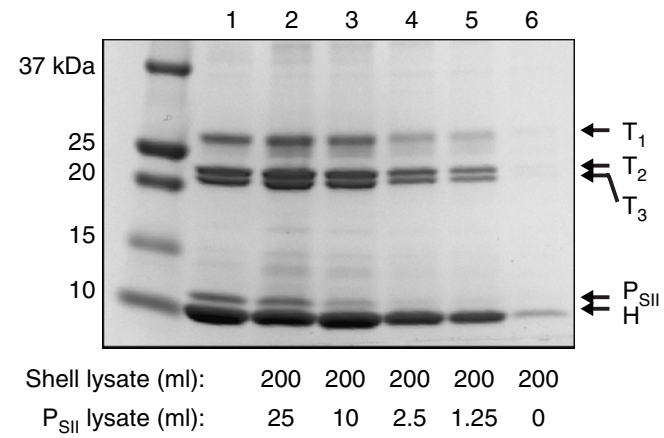

g

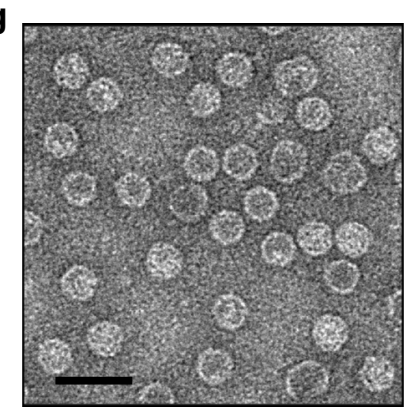

Fig. 1 Comparison of different shell preparation methods. a SDS-PAGE analysis of HO shell preparations. Lane 1: Classic, Lane 2: in vivo capped, Lane 3: ex vivo capped. Shell protein identities indicated by arrows; loading normalized to A280 readings. b-d Negative stain TEM micrographs of three different shell preparations and corresponding structural models. Scale bar $=100 \mathrm{~nm}$. e SDS-PAGE analysis of eluates from pentamer titration experiment. Lane 1: Ex vivo capped shells from Fig. 1a, Lanes 2-6: Titration of pentamers with equivalent volumes of each culture used in mixing experiments expressed in milliliters. $\mathbf{f}$ SDS-PAGE analysis of Halo carboxysome shell preparation using affinity purification. $\mathbf{g}$ Negative stain TEM micrographs of Halo carboxysome shell preparation. Scale bar $=50 \mathrm{~nm}$. Results presented are representative of two independent biological replicates for a-d, $\mathbf{f}$, and $\mathbf{g}$. Findings in figure (e) recapitulate pilot experiments in which a subset of specified lysate ratios were used

pure whereas both CAP-purified shell preparations are $>95 \%$ pure and the entire protocol from cell pellets to shells can be performed in a matter of hours. In addition, the final yield of shells for the different methods is similar, ranging from 0.40 to $0.55 \mathrm{mg}$ purified shell per gram cell pellet. Control reactions using a pentamer variant without the Strep-II tag fail to appreciably recover shells during the ex vivo CAP protocol, indicating that the method is dependent on specific interactions with the affinity resin (Supplementary Fig. 3c).

Analysis of the initial ex vivo capping experiment suggested that at the 1:2(v/v) mixing ratio of pentamer lysate to shell lysate used, the pentamers are present in stoichiometric excess (i.e., greater than 12 pentamer subunits per shell particle). This is evidenced by a substantial peak comprised primarily of pentamer protein which elutes prior to the capped shells during the anion-exchange chromatography run (Supplementary Fig. 3b). While we cannot be certain at this stage that every vertex is occupied when pentamers are in such excess, we nonetheless performed a pentamer titration to determine whether putative substoichiometric amounts of pentamer provide sufficient avidity to pull-down the (presumably) partially capped shells. Equal volumes of shell lysates were mixed with a range of pentamer lysate amounts (as low as 1:160 pentamer to shell volumetric mixing) and CAP-purified. The eluates were subjected to
SDS-PAGE analysis, this time without anion-exchange polishing steps in order to preserve the relative ratios of all eluted proteins. As shown in Fig. 1e, decreasing pentamer to hexamer band intensities in tandem with decreased pentamer loading indicate that sub stoichiometric amounts of pentamer are still able to recover shells and overall impact on shell yield is modest above a certain pentamer loading threshold. We attribute the trace amounts of shells recovered in the no-pentamer control lane to incomplete elution of pentamers and/or shells from previous StrepTrap chromatography runs. Together, these results indicate that partially capped shells maintain sufficient avidity to be CAPpurified-a property that may be applied toward rapid purification of shells that can still be loaded with cargo ex vivo through the remaining pentamer vacancies and then fully capped.

Finally, we show that other shell systems are amenable to affinity-based purification by appending a Strep-II tag to the pentameric $\mathrm{CcmL}$ protein in a synthetic operon used to produce beta-carboxysomal shells from Halothece sp. PCC $7418^{19}$ (Halo hereafter). As with the in vivo CAP described above, proteins were heterologously expressed in E. coli and purified by StrepTrap and anion-exchange. Figures 1f, g reveals that highly pure, morphologically homogeneous particles are obtained via affinity purification. In the native systems, the absence of $\mathrm{CcmL}$ typically results in elongated carboxysomes; the presence of 
pentamer seems to be required for the termination of facet growth $^{10,25}$. Accordingly, uncapped synthetic carboxysome shells cannot be complemented in trans as in the ex vivo CAP method used for HO shells. Nevertheless, the rapid isolation of BMCs of a completely different type suggests the method may be broadly applicable to other shells systems.

CAP facilitates screening of shell formation to define a minimal shell. Recent work in our lab ${ }^{2}$ revealed $\mathrm{HO}$ shells to be $\mathrm{T}=9$ icosahedra with one of the three possible BMC-T $\left(\mathrm{T}_{1-3}\right)$ proteins occupying the central position of each facet. Due to high shape complementarity between the trimers despite significant primary sequence divergence, we predicted that the trimers would be interchangeable. We therefore employed CAP to screen for the potential formation of shells comprised of the hexamer, one of the three trimers, and the pentamer protein. Shells were produced by expression from three plasmids each bicistronically expressing the hexamer and a single trimer. Because we were employing this method as a facile screen for shell assembly, we omitted subsequent anion-exchange polishing steps. Consequently, by ensuring $\mathrm{P}_{\mathrm{SII}}$ protein is in stoichiometric excess and equally applied to each $\mathrm{HT}_{\mathrm{n}}$ lysate, densitometric analysis of the $\mathrm{P}_{\mathrm{SII}}$ to hexamer band-intensity ratios gives a crude measure of relative shell yields (SDS-PAGE samples are loaded based on normalized A280 values).

As shown in Fig. 2a, expression of any single BMC-T domain with BMC-H is sufficient to form shells as assayed by CAP. To our knowledge, shell formation from just a single species each of hexamer, trimer and pentamer proteins has not previously been observed. Because identical trimers occupy each facet in these minimal shells, the method provides a means of isolation of BMC shells that are molecularly defined icosahedra. Interestingly, the $\mathrm{P}_{\mathrm{SII}}$ to $\mathrm{BMC}-\mathrm{H}$ ratios reveal notable differences between the band intensities of the three shell preparations. Whereas shells comprised of just $\mathrm{T}_{1}$ and $\mathrm{T}_{3}$ trimers $\left(\mathrm{HT}_{1} \mathrm{P}_{\mathrm{SII}}\right.$ and $\mathrm{HT}_{3} \mathrm{P}_{\mathrm{SII}}$, respectively) show similar pentamer:hexamer ratios (lanes 1 and 3 ), the yield of $\mathrm{HT}_{2} \mathrm{P}_{\text {SII }}$ shells is markedly reduced as indicated by a lower hexamer:pentamer band-intensity ratio. Whether this is attributable to the impact a particular BMC-T has on shell assembly efficiency or is merely an artifact of differential expression/stability between the trimers remains undetermined. We note no major morphological differences in these minimal shell preparations, further reinforcing the notion that each individual trimer serves identical structural roles within the context of the shell architecture, despite their presumed differences in permeability (Fig. 2b, d).

EnCO enables robust encapsulation of cargo. To date, published encapsulation methods for heterologous cargo have been inefficient ${ }^{19-22}$. We sought a synthetic route to encapsulation that circumvents the shortcomings of EPs by using the SpyTag/SpyCatcher split bacterial adhesin system ${ }^{26}$. This technology relies on genetically fusing a $9 \mathrm{kDa}$ SpyCatcher domain to one protein partner and a $1.4 \mathrm{kDa}$ (13 residue) SpyTag to another protein partner. When the SpyCatcher and SpyTag polypeptides encounter one another, an isopeptide bond autocatalytically forms between them, covalently linking the two partner proteins. We reasoned that a SpyCatcher or SpyTag localized to the lumen of a BMC shell would serve as a defined docking site for cargo protein tagged with SpyTag or SpyCatcher (respectively).

$\mathrm{T}_{1}$ was chosen as a target for insertional mutagenesis of the SpyTag and SpyCatcher sequences. As shown in Fig. 3a, a region was identified on the lumenal side between alpha-helix two and beta-strand four, which is not predicted to make any contacts with neighboring shell proteins and which lies $\sim 24 \AA$ from the subunit's central pore (the entire subunit is $\sim 70 \AA$ edge-to-edge). This spacing from the threefold symmetry axis of the subunit mitigates the possibility of steric clashes from inserted/conjugated domains which could prevent the assembly of individual protomers into the trimeric subunit and/or result in incomplete cargo conjugation. SpyCatcher and SpyTag, flanked by six residue glycine-serine linkers, were inserted into $\mathrm{T}_{1}$ to create $\mathrm{T}_{\mathrm{SC}}$ and $\mathrm{T}_{\mathrm{ST}}$ constructs, respectively (Fig. $3 \mathrm{~b}, \mathrm{c}$ ).

The wild-type $T_{1}$ was replaced with the $\mathrm{T}_{\mathrm{SC}}$ and $\mathrm{T}_{\mathrm{ST}}$ mutants in plasmids that express the full complement of trimers $\left(\mathrm{pHT}_{1} \mathrm{~T}_{2} \mathrm{~T}_{3}\right)$ as well as the minimal shell producing $\mathrm{pHT}_{1}$ plasmid to create,

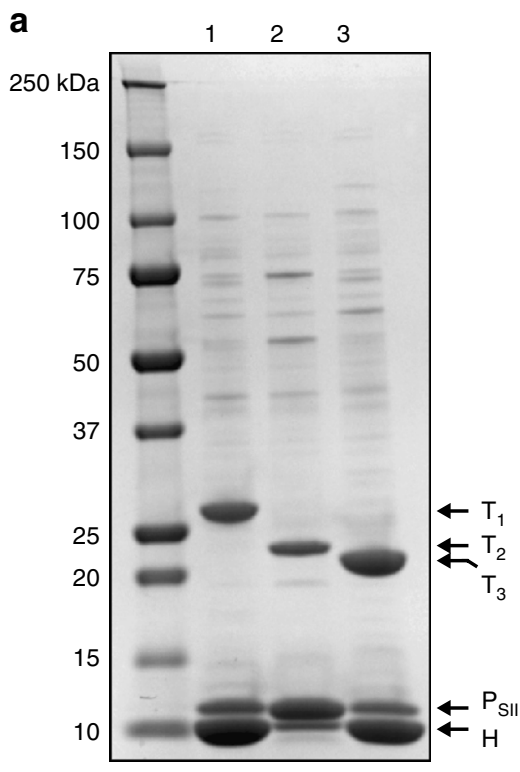

b
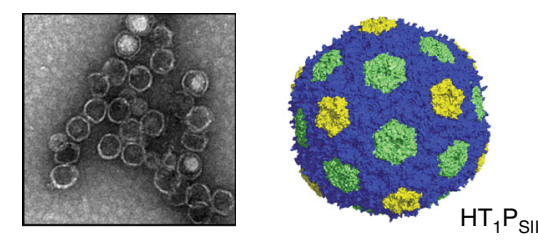

C

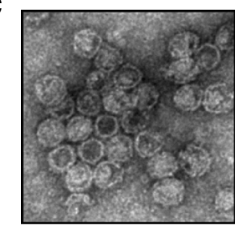

d

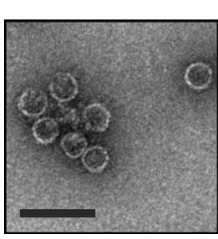

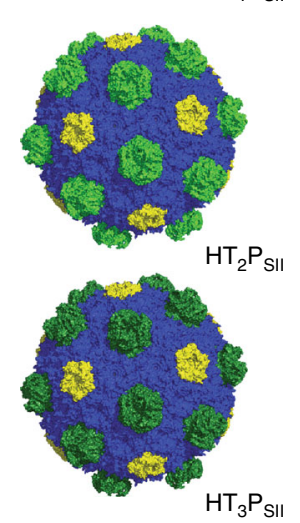

Fig. 2 Comparison of different minimal shells. a SDS-PAGE analysis of crude shell preparations. Lane 1: $\mathrm{HT}_{1} \mathrm{P}_{\text {SII }}$ Lane 2: $\mathrm{HT}_{2} \mathrm{P}_{\text {SII }}$ Lane 3: $H T_{3} \mathrm{P}_{\text {SII }}$. Shell protein identities indicated by arrows. b-d Negative stain TEM micrographs of three minimal shell preparations and corresponding structural models. Scale bar $=$ $100 \mathrm{~nm}$ 
a

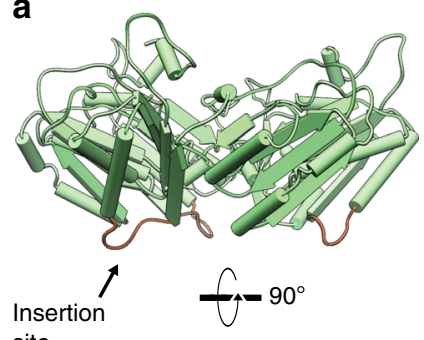

site

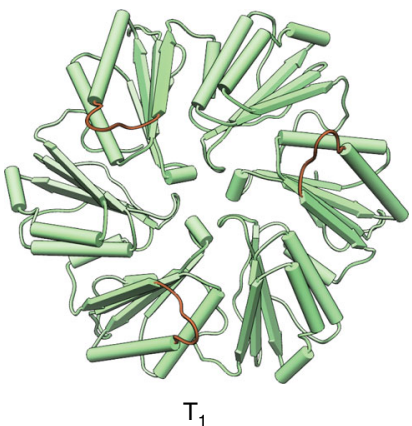

b

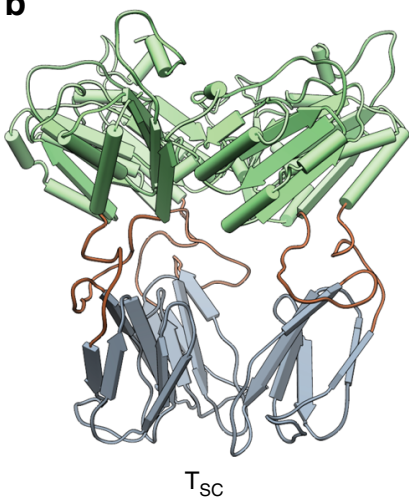

C

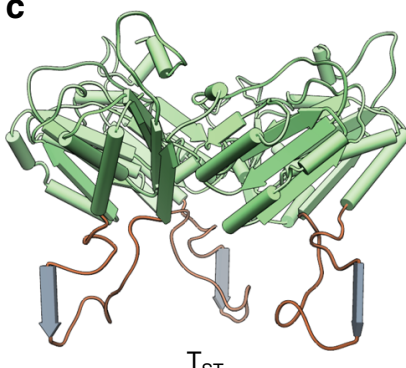

Fig. 3 Molecular models of $T_{1}, T_{S C}$ and $T_{S T}$ subunits. a Model of $T_{1}$ (wt) (PDB: $5 \mathrm{DIH}$ ) as viewed from side of shell (top) and lumen (bottom). $\mathbf{b}$ and c Models of $\mathrm{T}_{\mathrm{SC}}$ and $\mathrm{T}_{\mathrm{ST}}$ (respectively) viewed from the side. Flexible coil regions colored in brown; SpyCatcher and SpyTag regions colored in gray

respectively, $\mathrm{pHT}_{\mathrm{SC}} \mathrm{T}_{2} \mathrm{~T}_{3} / \mathrm{pHT}_{\mathrm{SC}}$ and $\mathrm{pHT}_{\mathrm{ST}} \mathrm{T}_{2} \mathrm{~T}_{3} / \mathrm{pHT}_{\mathrm{ST}}$. SpyTag and SpyCatcher was appended to the $\mathrm{N}$-terminus of the cyan fluorescent protein mTurquoise $2^{27}$ and cloned into a compatible and orthogonally inducible plasmid with a C-terminal hexahistidine tag (sTcfp).

Shell and cargo plasmids were co-transformed and shell expression was induced either with or without simultaneous induction of ${ }_{\mathrm{sT}} \mathrm{cfp}$ cargo. CAP was then used to isolate the resultant shells. SDS-PAGE analysis of unpolished eluates is shown in Fig. 4a. In contrast to the wild-type shells in lane 1 in which the wild-type $T_{1}$ protein is detected at its normal relative abundance, the $\mathrm{T}_{\mathrm{SC}}$ is undetectable in the putative $\mathrm{HT}_{\mathrm{SC}} \mathrm{T}_{2} \mathrm{~T}_{3} \mathrm{P}_{\mathrm{SII}}$ shells. However in lane 3 , the fused $\mathrm{T}_{\mathrm{SC}} \sim_{\mathrm{ST}} \mathrm{cfp}$ trimer, which migrates at $\sim 75 \mathrm{kDa}$, is observed-likely due to the increase in the protein mass upon conjugation to cargo allowing the fusion to exceed the stain's limit of detection. This would suggest that the $\mathrm{T}_{\mathrm{SC}}$ protein is at a modest competitive disadvantage for integration into shells compared to wild-type $T_{1}$ and $T_{2} / T_{3}$ and/or could result from reduced solubility of $\mathrm{T}_{\mathrm{SC}}$ relative to $\mathrm{T}_{1}$. Nevertheless, these $\mathrm{HT}_{\mathrm{SC}} \sim_{\mathrm{ST}} \mathrm{cfp} \mathrm{T}_{2} \mathrm{~T}_{3} \mathrm{P}_{\mathrm{SII}}$ shells exhibit normal morphology by microscopic analysis and have a fluorescence emission spectrum consistent with mTurquoise2 (Supplementary Figs. $4 \mathrm{a}, \mathrm{b}$ ), thus proving that cargo-fused $\mathrm{T}_{\mathrm{SC}}$ can integrate into shells without any gross morphological disruption. Furthermore, whereas EP-based encapsulation in the HO shell system (among others) requires western blotting to detect cargo, the detection of loaded cargo by coomassie stain alone represents a significant improvement over existing methods.

Minimal shells harboring only the $\mathrm{T}_{\mathrm{SC}}$ subunit have more cargo binding capacity due to the absence of competition for the trimer subunit position with $\mathrm{T}_{2}$ and $\mathrm{T}_{3}$ proteins-a theoretical 60 copies of cargo could be recruited by the twenty trimeric subunits. This was confirmed by characterizing cargo loading using the $\mathrm{pHT}_{\mathrm{SC}}$ plasmid and results are shown in Fig. 4a lanes 4-6. Lane 5, in which

expression of cargo is not induced, now reveals the presence of $\mathrm{T}_{\mathrm{SC}}$ as expected (lest no shells be formed) and lane 6 shows the $T_{S C}$ band is nearly completely converted to the cargo-fused $\mathrm{T}_{\mathrm{SC}} \sim \mathrm{ST} \mathrm{Cfp}$ form in these shell preparations. Interestingly, a small amount of free ${ }_{\text {ST }} \mathrm{cfp}$ co-purifies with the shells (lane 6) which could be a result of stochastic encapsulation or proteolytic deconjugation from $\mathrm{T}_{\mathrm{SC}}$. Western blotting against the hexahistidine tag present in ${ }_{\mathrm{ST}} \mathrm{cfp}$ confirms the identity of these proteins and reveals that the faint band at $\sim 75 \mathrm{kDa}$ in lane 5 is a contaminant and not $\mathrm{T}_{\mathrm{SC}} \sim \mathrm{ST} \mathrm{Cfp}$ which could result from leaky expression of the ${ }_{\mathrm{ST}} \mathrm{cfp}$ in the absence of inducing agent (Supplementary Fig. 4c).

When SpyTag is inserted into $\mathrm{T}_{1}$ and SpyCatcher-fused to cargo (i.e. the inverse arrangement), similar behavior is observed in the context of full shells: the $\mathrm{T}_{\mathrm{ST}} \sim_{\mathrm{SC}} \mathrm{Cfp}$ fusion is detected but not at the relative abundance expected in wild-type $\mathrm{HT}_{1} \mathrm{~T}_{2} \mathrm{~T}_{3} \mathrm{P}_{\text {SII }}$ shells (Fig. $4 \mathrm{~b}$ lane 1). However, in comparison to the minimal $\mathrm{HT}_{\mathrm{SC}} \sim \mathrm{ST}_{\mathrm{T}} \mathrm{CfP}_{\mathrm{SII}}$ shells in Fig. 4a lane 6, a significant fraction of the $\mathrm{T}_{\mathrm{ST}}$ subunit remains unconjugated to the $\mathrm{sC} \mathrm{cfp}$ cargo (Fig. $4 \mathrm{~b}$ lane 2). This could indicate that the $\mathrm{T}_{\mathrm{ST}}$ is less efficient at recruiting cargo compared to $\mathrm{T}_{\mathrm{SC}}$ and/or that SpyCatcher-fused cargo is sterically frustrated from productive binding to the $T_{S T}$ subunit. A final, non-mutually exclusive scenario is that shells form prior to cargo recruitment and the larger ${ }_{\mathrm{sc}} \mathrm{cfp}(42.7 \mathrm{kDa})$ is less able to transit the pentamer vacancies compared to ${ }_{\mathrm{ST}} \mathrm{cfp}$ $(29.9 \mathrm{kDa}) . \mathrm{HT}_{\mathrm{ST}{ }_{\mathrm{SC}} \mathrm{CfpP}}$ SII shells are obtained at levels similar to $\mathrm{HT}_{1} \mathrm{~T}_{2} \mathrm{~T}_{3} \mathrm{P}_{\text {SII }}$ shells $(0.40 \mathrm{mg}$ per gram of cell pellet) while $\mathrm{HT}_{\mathrm{SC} \sim \mathrm{ST}} \mathrm{CfpP}_{\mathrm{SII}}$ shells incur a significant reduction in yield (0.04 mg per gram cell pellet), though purity is comparable after anion-exchange chromatography is performed (Supplementary Fig. 5a). Finally, we calculated the number of cfp cargo proteins per shell using fluorescence intensity values and find that $\mathrm{HT}_{\mathrm{SC}} \sim{ }_{\mathrm{ST}} \mathrm{cfp} \mathrm{P}_{\mathrm{SII}}$ shells have $\sim 73$ copies per shell and $\mathrm{HT}_{\mathrm{ST}} \sim \mathrm{sC}$ cfpP $\mathrm{P}_{\mathrm{SII}}$ shells have $\sim 58$ copies per shell (Supplementary Fig. 5b). These numbers are in reasonable agreement with theoretical estimates based on percent conversion of the trimers to their respective conjugates as observed in SDS-PAGE analysis.

Based on the assembly principles and structure of $\mathrm{HO}$ shells reported by Sutter et al. ${ }^{2}$, the minimal shells in lanes 4-6 harbor 20 trimer subunits, nearly all of which are conjugated to cargo in the $\mathrm{HT}_{\mathrm{SC}} \sim{ }_{\mathrm{ST}} \mathrm{Cfp} \mathrm{P}_{\mathrm{SII}}$ shell preparations according to the aforementioned SDS-PAGE and fluorescence analysis. This should increase the apparent shell thickness and indeed, examination of transmission electron micrographs of the minimal shell compositions depicted in Fig. 4a lane 6 reveal notable differences in shell appearance: cargo-loaded shells appear thicker at $\sim 6.2 \mathrm{~nm}$ and more stippled compared to $\mathrm{HT}_{1} \mathrm{P}_{\text {SII }}$ shells (Fig. $2 \mathrm{~b}, \sim 4.7 \mathrm{~nm}$; measurements are mean of $n=3$ discrete shells) as well as empty $\mathrm{HT}_{\mathrm{SC}} \mathrm{P}_{\text {SII }}$ shells (Supplementary Fig. 6; see Supplementary Fig. 7 for shell measurements). Together, these findings validate EnCo as a robust method for encapsulation of cargo into morphologically undisrupted shells using trimer subunits functionalized with molecular traps.

Multiple cargo proteins can be loaded ex vivo at defined ratios. The in vivo encapsulation results do not allow us to distinguish between two non-mutually exclusive scenarios: $\mathrm{T}_{\mathrm{SC} / \mathrm{ST}} \sim \mathrm{cargo}$ conjugation occurs prior to integration into proto-shells, or empty shells form first and cargo then conjugates to the trimer subunits after entry through the pentamer holes. The pentamer vacancies are $\sim 47 \AA$ diameter and can accommodate mTurquoise2 (Supplementary Fig. 8a). We tested whether cargo could be loaded into pre-formed shells ex vivo in biological duplicates by mixing clarified lysates of strains expressing $\mathrm{HT}_{\mathrm{SC}}$ shells with clarified lysates containing the previously described $\mathrm{ST}_{\mathrm{T}} \mathrm{cfp}$ as well as SpyTagged yellow fluorescent protein, SYFP2 ${ }^{28}\left(\mathrm{sT}_{\mathrm{yfp}}\right)$ at 
a

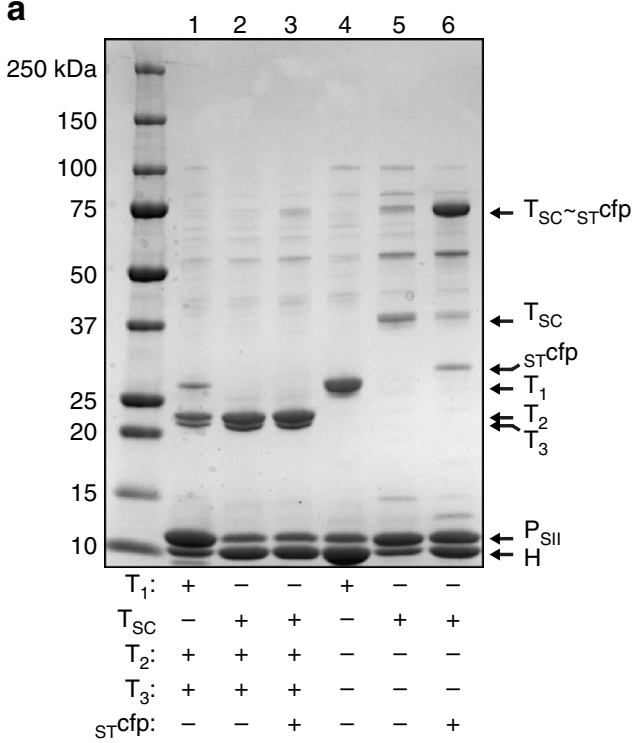

b

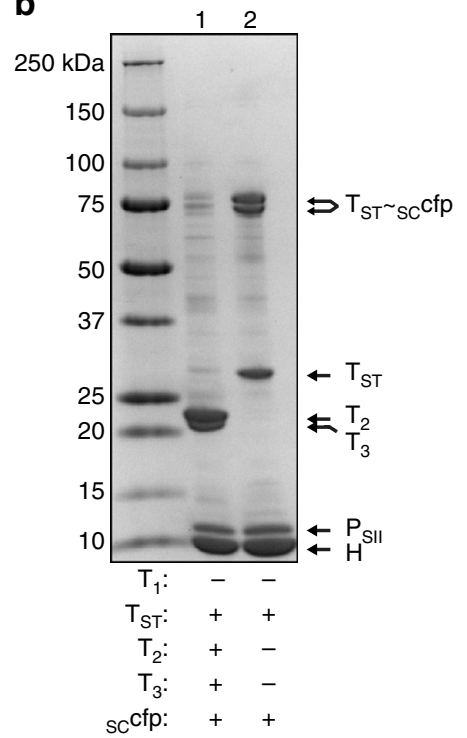

C
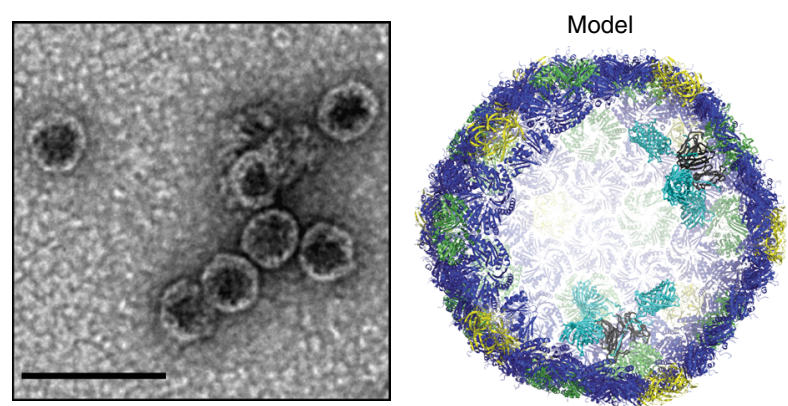

Fig. 4 SDS-PAGE and electron micrographs of various shell preparations. a, b SDS-PAGE of shell preparations. Composition of shell preparations given in tabular form below each lane. c Negative stain TEM of $\mathrm{HT}_{\mathrm{SC} \sim \mathrm{ST}} \mathrm{cfpP}_{\mathrm{SII}}$ shell preparation (lane 6 in figure $\mathbf{a}$ ) and cutaway model of shells (cfp rendered in turquoise, not completely functionalized for clarity). Results presented are representative of at least two independent biological replicates of each sample preparation

varying volumetric ratios. These two fluorophores are spectrally well-matched as a Förster resonance energy transfer (FRET) pair $^{27}$ and therefore their co-localization is detectable spectrophotometrically. Expression under identical conditions in separate strains results in the same abundance of the ${ }_{\mathrm{ST}} \mathrm{cfp}$ and ${ }_{\mathrm{ST}} \mathrm{yfp}$ proteins in whole cell lysates (Supplementary Fig. 8b).

After cargo loading, the mixtures were subjected to CAP and analyzed spectrophotometrically; flow-through fractions which contain unencapsulated ${ }_{\mathrm{ST}} \mathrm{cfp} / \mathrm{sT} \mathrm{yfp}$ were also collected for comparison. Shells were excited at $405 \mathrm{~nm}$ (a wavelength capable of ${ }_{\mathrm{sT}} \mathrm{cfp}$ excitation with negligible excitation of ${ }_{\mathrm{sT}} \mathrm{yfp}$ ) and emission spectra were collected from 450 to $600 \mathrm{~nm}$. As shown in Fig. 5a, increasing the proportion of sтyfp in the mixture attenuates the emission of ${ }_{\mathrm{ST}} \mathrm{cfp}$ (emission maximum $474 \mathrm{~nm}$ ) with a concomitant increase in emission from sт yfp (emission maximum $527 \mathrm{~nm}$ ) indicating FRET and co-localization of the two fluorophores. Comparison of the fluorescence intensities of the two fluorophores in both the shell preparations and flowthrough fractions corroborate these emission scan data (Supplementary Fig. 8d). These data demonstrate that ${ }_{\mathrm{ST}} \mathrm{cfp}$ and ${ }_{\mathrm{ST}} \mathrm{yfp}$ соlocalization is mediated by co-encapsulation within $\mathrm{HT}_{\mathrm{SC}}$ shells and that multi-cargo relative abundances can be easily programmed via ex vivo encapsulation-an important consideration for optimizing flux through multi-enzyme cascades and which is not readily achievable in vivo without laborious optimization of cargo expression levels. Finally, the encapsulation efficiency of ex vivo cargo loading for these $\mathrm{HT}_{\mathrm{SC} \sim{ }_{\mathrm{ST}}} \mathrm{cfpP}_{\mathrm{SII}}$ shells was assessed by SDS-PAGE analysis along with the inverted architecture $\left(\mathrm{HT}_{\mathrm{ST}} \sim{ }_{\mathrm{SC}} \mathrm{cfpP} \mathrm{P}_{\mathrm{SII}}\right.$ shells). As shown in Fig. 5b, c, the amount of unconjugated $\mathrm{T}_{\mathrm{SC}}$ and $\mathrm{T}_{\mathrm{ST}}$ is similar, if not lower compared to the in vivo encapsulation experiments (Fig. 4a lane 6 and $4 \mathrm{~b}$ lane 2). Because these ex vivo-loaded shells are formed prior to the introduction of cargo, this indicates that the observed relative encapsulation efficiency of the two EnCo architectures is not due to the different sizes of cargo transiting through the pentamer gaps. Control experiments in which incompatibly-tagged cargo was tested for encapsulation (e.g., using sccfp with $\mathrm{HT}_{\mathrm{SC}}$ shells) reveals non-specific stochastic encapsulation is minor (Supplementary Fig. 8e).

Interrogation of HO shell permeability using a FRET-based sensor. In comparison to storage-based nanocompartments like viruses and ferritins ${ }^{29}$, BMC function depends on selective permeability to an array of molecules-the identities of which depend on the particular native physiological function of a given BMC (recently reviewed in ref. ${ }^{30}$ ). The uncapped HO shells admit macromolecular species yet should prevent their entry/egress when fully capped. To test this behavior and confirm that CAP results in fully complemented shells, we developed a FRET-based permeability probe and apply it towards elucidating the permeability of HO shells to protein and small molecule size scales. The tobacco etch virus protease (TEVp) cleavage site ${ }^{31}$ followed by a six-residue tetracysteine motif was inserted between the 

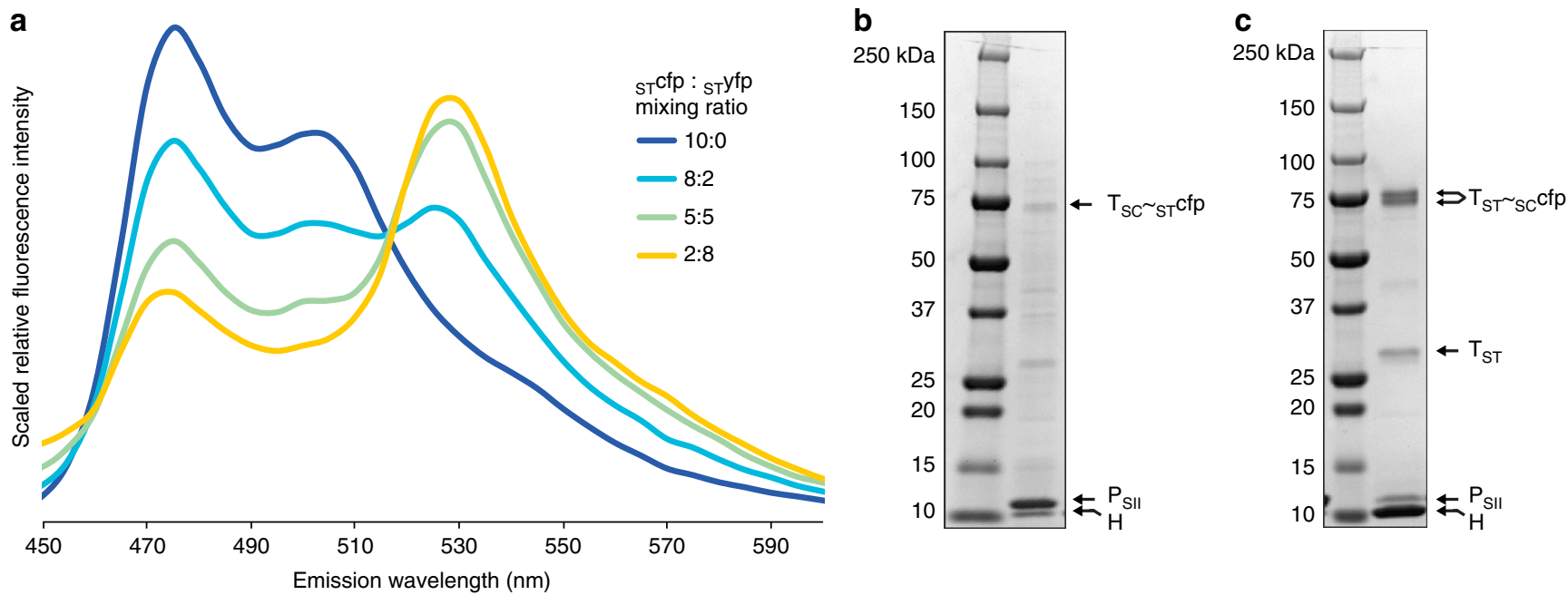

Fig. 5 Fluorescence spectra and SDS-PAGE analysis of shells containing ex vivo programmed cargo. a Scaled emission spectra (excitation: 405 nm) of programmed cargo. sтyfp-only trace (0:10) was not plotted-in the absence of a FRET donor, the fluorescence signal is negligible. $\mathbf{b}$ SDS-PAGE analysis of sTcfp (10:0) programmed shells. c SDS-PAGE analysis of sccfp programmed shells. Results are representative of two independent biological replicates

C-terminus of mTurquoise 2 and the hexahistidine tag of the ${ }_{\mathrm{ST}} \mathrm{cfp}$ construct to create the SpyTagged permeability probe, sт probe. Tetracysteine motifs tightly and specifically bind conditionally fluorescent biarsenical dyes such as the fluorescein-derived $\mathrm{FlAsH}$ reagent $^{32}$. At $664.5 \mathrm{Da}$ and $\sim 10 \AA$ at its narrowest point, the FlAsH reagent is in a similar size regime as cofactors like NADPH (744.4 Da) and therefore may be admitted through some subunit pores-likely one or more trimers, as the hexamer's $\sim 6.8 \AA$ pore is too small to accommodate the FlAsH reagent ${ }^{5,33,34}$. The spectral properties of FlAsH are similar to SYFP2; binding to stprobe positions the fluorophore within the Förster distance of mTurquoise 2 and therefore this event can be detected spectrophotometrically. The spectral shift can subsequently be reversed by proteolytic removal of the labeled tetracysteine motif using TEVp. stprobe's design and spectrophotometric behavior in response to incubation with $\mathrm{FlAsH}$ and TEVp when unencapsulated are depicted in Fig. 6a, b. Because TEVp also removes the $\mathrm{C}$-terminal hexahistidine tag from the probe, cleavage can also be detected using western blotting against this epitope. SDS-PAGE analysis of the final probe preparation and western blotting confirm that, as expected, the BMC-P protein itself has no inhibitory activity on TEVp (Supplementary Fig. 9a, b).

We performed a classical shell preparation of sT probe-loaded, uncapped shells $\left(\mathrm{HT}_{\mathrm{SC}} \sim \mathrm{ST}_{\mathrm{T}}\right.$ probe $\left.\mathrm{T}_{2} \mathrm{~T}_{3}\right)$. Addition of the FlAsH reagent to the shells results in a fluorescence emission shift which is reverted upon TEVp addition (Fig. 6c, top row), recapitulating the behavior of unencapsulated probe. This demonstrates, as expected, that FlAsH and TEVp ( $\sim 36 \AA$ in diameter) can enter the shells and proteolyze the encapsulated probe. We next show that shells pre-capped via incubation with purified pentamer $\left(\mathrm{HT}_{\mathrm{SC} \sim \mathrm{ST}}\right.$ probe $\left._{2} \mathrm{~T}_{3} \mathrm{P}_{\mathrm{SII}}\right)$ still undergo a spectral shift when FlAsH is added, yet show no reversion in spectral emission upon addition of TEVp (Fig. 6c, bottom row). Exclusion of TEVp from these capped shells is confirmed by western blot analysis (Supplementary Fig. 9d). We conclude that addition of the pentamer to uncapped shells is necessary and sufficient to preclude the entry of TEVp, though interestingly, these capped shells remain permeable to the $\mathrm{FlAsH}$ reagent, indicating it may be entering through the pores of one or more subunits. By leveraging EnCo for encapsulation of a permeability probe, these data indicate that CAP yields fully complemented shells with no unoccupied pentamer positions.

\section{Discussion}

In this study, we have elaborated the function of bacterial microcompartment shell proteins through the introduction of affinity handles and molecular traps. Complementation-based affinity purification (CAP) enables rapid purification of shells and by extension, screening for the formation of particles comprising varying subunits. Encapsulation via covalent-linkage (EnCo) allows facile programming of cargo composition expressed in cis and in trans. Using a permeability probe, we unambiguously demonstrate that shells capped in trans are fully complemented barriers against the transit of a macromolecular species (TEV protease) and yet admit the small molecule, FlAsH-likely through one or more of the distinct types of pores found in the shell proteins.

Previous work has shown that other BMCs such as the carboxysome can form without pentamers, though aberrant structures were observed microscopically in cells ${ }^{6,10,25}$. In contrast, we observe no structural polymorphisms in $\mathrm{HO}$ shell preparations when pentamers are absent. Moreover, the isolation of morphologically undisrupted minimal shells, each harboring a single trimer species, implies redundancy at the structural level while differing pore and stacking configurations implicate nonredundant functions in selective molecular transport across the shell. The ability to form uncapped shells enables the encapsulation of cargo ex vivo. As demonstrated, this allows multiple cargo species (which need not be expressed in the shell host) to be introduced into the shells at programmed ratios. Trapping SpyTag/SpyCatcher-fused cargo in shells via EnCo is simple and efficient; however in principle any species able to transit the $47 \AA$ diameter pentamer gap could be captured via co-incubation and capping. Such stochastic encapsulation would be of particular use in encapsulating molecules which cannot be tagged (e.g., acylCoA substrates), or even wholly abiotic materials.

Although there is a report of affinity enrichment of bacterial microcompartments ${ }^{35}$, the resultant particles were heterogeneous and morphologically aberrant. This may be attributable to appending hexahistidine tags to multiple shell proteins which may sterically frustrate proper assembly. In contrast, the lack of any pentamer-pentamer subunit interactions in assembled shells advantages the use of this building block for embelishments and we have demonstrated that very few Strep-II tags per particle provide sufficient avidity to pull-down highly pure shells. The 
a

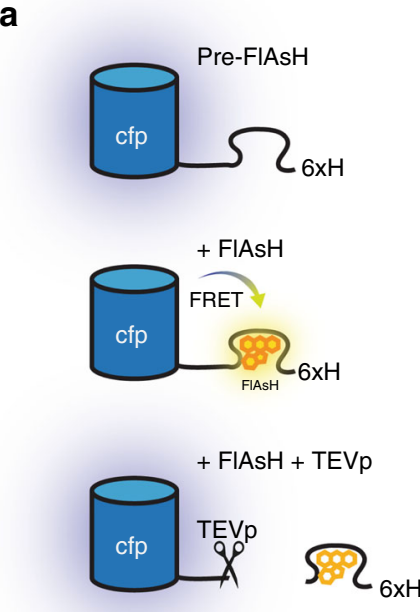

\section{b}
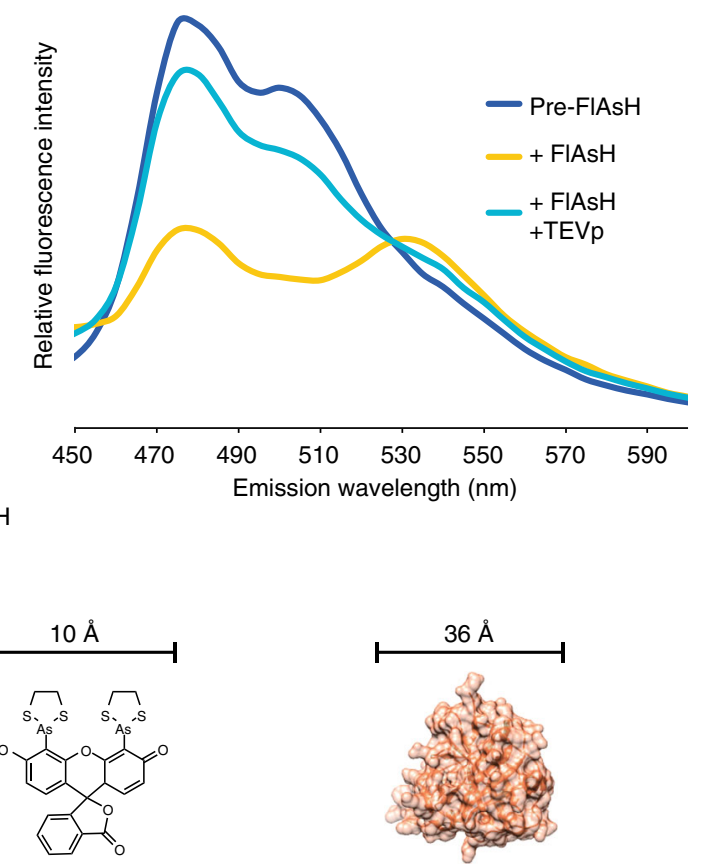

C

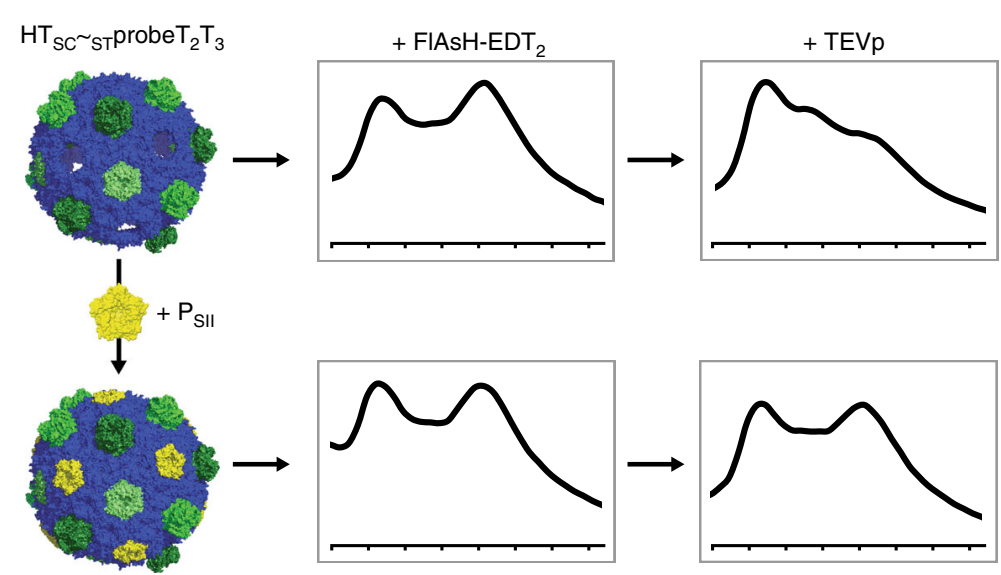

$\mathrm{HT}_{\mathrm{SC}} \sim \mathrm{ST}$ probeT $\mathrm{T}_{2} \mathrm{P}_{\mathrm{SII}}$

Fig. 6 Probe properties and permeability assay of uncapped and capped shells. a Schematic of probe behavior in presence of FIAsH and TEV protease. b Fluorescence emission spectra (excitation: $405 \mathrm{~nm}$ ) of unencapsulated probe. c Emission spectra (450-600 nm) of encapsulated probe in uncapped and capped shells, in the presence of FIAsH and TEV protease. Axis numbers omitted for clarity; tick marks correspond to numbers in $\mathbf{b}$. Results are representative of two independent technical replicates. Bacterial microcompartments are protein-bound organelles encapsulating segments of metabolic pathways. Here the authors functionalize shell proteins to facilitate facile purification and enable cargo encapsulation via covalent linkage

isolation of beta-carboxysomal (Halo) shells demonstrates that this affinity pull-down strategy is applicable to other microcompartments that frequently exhibit morphological heterogeneity. Given the homology among the shell proteins of all BMCs, we expect that this method, after identifying specific combinations of subunits to form synthetic shells, will be generally applicable to other BMC systems.

The observations by us and others that encapsulation peptides are often ineffective at recruiting heterologous cargo to the lumen of BMC shells motivated us to pursue alternative means of encapsulation. Strategies for nanoparticle encapsulation such as engineering complementary electrostatics have proven useful for other nanocontainers such as lumazine synthase ${ }^{36}$. However, this approach is not targeted and may non-specifically package resident charged molecules (e.g., nucleic acids) when performed in vivo. The SpyTag/SpyCatcher system has been used for encapsulation in other nanocompartments such as MS2 viral capsids and excitingly, encapsulated enzymes were shown to be more robust ${ }^{37}$. However MS2 viral capsids are composed of a single protein type and therefore lack any potential for compositional plasticity which may decrease their utility. We believe that shared architectural features and structurally homologous insertion sites will allow translation of the SpyCatcher/SpyTag system to other BMC types. Moreover, despite the demonstrated superiority of EnCo to existing technology, improvements may be realized with additional protein engineering. For example 
alternatives to the currently employed poly-G/S linkers that flank SpyTag/SpyCatcher could improve solubility, cargo recruitment and integration into shells. Additionally, because expression and shell formation dynamics remain unexplored, in vivo metabolic engineering applications will require empirical testing of induction timing and strength to optimize loading efficiency.

While numerous studies have demonstrated that the permeability of BMCs can be altered via rational mutagenesis of porelining residues, or even whole-sale swapping of subunits from one BMC system to another ${ }^{38-40}$, assessing BMC permeability to a given small molecule is non-trivial, much less elucidating the permeability of specific subunits to said molecule. Our fluorescence-based probe serves as proof-of-principle for the use of these shell protein functionalizations for assessing shell permeability to small molecules in real time. In principle, any encapsulated enzymatic transformation(s) that results in a spectroscopic signal could serve as a readout of shell permeability, enabling the characterization of shell permeability to physiologically relevant species such as $\mathrm{NAD}(\mathrm{P}) \mathrm{H}$. Leveraging the plasticity of subunit composition combined with altered selectivity from pore mutagenesis could allow for the facile creation of shells with bespoke permeabilities-likely a vital step toward increasing efficiencies of encapsulated, non-native pathways. The development of the EnCo and CAP methods represent significant steps towards more accessible physiological studies as well as realizing the biotechnological promises of bacterial microcompartments.

\section{Methods}

Shell expression. BL21(DE3) strains harboring shell plasmids were grown to OD600 $0.6-0.8$ in lysogeny broth at $37^{\circ} \mathrm{C}$ with $100 \mu \mathrm{g} / \mathrm{mL}$ ampicillin and induced with $50 \mu \mathrm{M}$ IPTG with a brief cold-shock on ice. When co-expressing SpyTag/ SpyCatcher cargo or $\mathrm{P}_{\mathrm{SII}}$, strains were additionally cultured with $50 \mu \mathrm{g} / \mathrm{mL}$ kanamycin and induced simultaneously with $5 \mathrm{ng} / \mathrm{mL}$ anhydrotetracycline. Incubation was then continued at $18^{\circ} \mathrm{C}$ for $16-20 \mathrm{~h}$.

Classical shell purifications. Shells were prepared using methods described in ref. ${ }^{2}$. Briefly, the cell pellet from a $2 \mathrm{~L}$ culture expressing $\mathrm{pHT}_{1} \mathrm{~T}_{2} \mathrm{~T}_{3}$ was resuspended in Tris-buffered saline $(20 \mathrm{mM}$ Tris, $50 \mathrm{mM} \mathrm{NaCl}$, pH 7.4 TBS 20/50 hereafter), lysed by French press and clarified by centrifugation at $25,000 \times g$ for $30 \mathrm{~min}$. After sucrose cushion and sucrose gradient centrifugations, shells were further purified by application to a MonoQ 10/100 GL anion-exchange column connected to an Äkta Pure system and elution with a shallow sodium chloride gradient (200-400 mM NaCl over 10 column volumes). Shell containing fractions were pooled and concentrated with a $15 \mathrm{~mL} 100 \mathrm{kDa}$ MWCO filter (Amicon) and stored on ice after the addition of $0.02 \%$ sodium azide as a preservative. For $\mathrm{HT}_{\mathrm{SC} \sim \mathrm{ST}}$ probe $\mathrm{T}_{2} \mathrm{~T}_{3}$ shells, classically purified $\mathrm{HT}_{\mathrm{SC}} \mathrm{T}_{2} \mathrm{~T}_{3}$ shells were incubated overnight with an excess of purified stprobe (see Supplementary Methods/ Expression and purification of ${ }_{\text {ST }}$ probe protein) at $4{ }^{\circ} \mathrm{C}$ and then excess sTprobe was removed via application to a HisTrap column, followed by another MonoQ step. All buffers for the $\mathrm{HT}_{\mathrm{SC} \sim \mathrm{ST}}$ probe $\mathrm{T}_{2} \mathrm{~T}_{3}$ shell preparation were amended with $5 \mathrm{mM}$ EDTA and $5 \mathrm{mM}$ TCEP to maintain reducing conditions.

Complementation-based affinity purification. For initial in vivo and ex vivo capping, cell pellets from $1 \mathrm{~L} \mathrm{pHT} \mathrm{T}_{2} \mathrm{~T}_{3}$ cultures were lysed using BPER-II amended with recombinant lysozyme and benzonase according to manufacturer's recommendation and clarified by centrifugation as above. Shell lysates were then mixed with clarified $\mathrm{P}_{\mathrm{SII}}$ lysates $(2: 1 \mathrm{v} / \mathrm{v}$ ratio of shell to pentamer pellets for $\mathrm{HT}_{1} \mathrm{~T}_{2} \mathrm{~T}_{3}$ shells; 10:1 for all minimal shells; see Supplementary Methods/Expression and purification of $\mathrm{P}_{\mathrm{SII}}$ protein) and incubated for $30 \mathrm{~min}$ to allow capping to occur. Lysates were then applied to a $5 \mathrm{~mL}$ StrepTrap (GE Healthcare) column equilibrated with Buffer A (100 mM Tris- $\mathrm{HCl}, \mathrm{pH} 7.4,150 \mathrm{mM} \mathrm{NaCl}, 1 \mathrm{mM}$ EDTA). The column was washed with six column volumes Buffer A and proteins were eluted in six column volumes Buffer B (20 mM Tris- $\mathrm{HCl}, \mathrm{pH} 7.4,50 \mathrm{mM}$ $\mathrm{NaCl}, 1 \mathrm{mM}$ EDTA, $2.5 \mathrm{mM} D$-desthiobiotin). Where specified, shells were further purified with anion-exchange chromatography and/or concentrated with $100 \mathrm{kDa}$ molecular weight cut-off Amicon spin filters as described. Capping experiments involving encapsulated cargo (Figs. 4,5 ) were scaled down to $0.2 \mathrm{~L}$ shell cultures and purified with $1 \mathrm{~mL}$ StrepTrap columns using otherwise identical methods.

Pentamer titration. Ex vivo capping was performed as described above except a range of clarified $\mathrm{P}_{\text {SII }}$ lysate amounts $(25,10,2.5,1.25$, and $0 \mathrm{~mL}$ equivalents) was added to $0.2 \mathrm{~L}$ equivalents $\mathrm{pHT}_{1} \mathrm{~T}_{2} \mathrm{~T}_{3}$ shell lysate.
Ex vivo loading of cargo into minimal shells. Cultures of $\mathrm{pET} 11 \mathrm{n}: \mathrm{HT} \mathrm{SC}_{\mathrm{ST}}$, pBbA2k:: ${ }_{\mathrm{ST} / \mathrm{SC}} \mathrm{mTurquoise2-6xHis}$ and $\mathrm{pBbA2k}: \mathrm{sT}_{\mathrm{ST}} \mathrm{SYFP} 2-6 \mathrm{xH}$ is were induced with $50 \mu \mathrm{M}$ IPTG (pET11n::HT ${ }_{\mathrm{SC} / \mathrm{ST}}$ ) or $50 \mathrm{ng} / \mathrm{mL}$ aTc (pBbA2k:: ${ }_{\mathrm{ST} / \mathrm{SC}}$ mTurquoise26xHis, pBbA2k:: ${ }_{\mathrm{ST}}$ SYFP2-6xHis). Biological replicates (total $n=2$ ) were performed with freshly re-transformed strains. Cell pellets were resuspended in Buffer A (4 mL per gram cell paste) supplemented with $12 \mu \mathrm{l}$ benzonase $(25 \mathrm{U} / \mu \mathrm{l})$, a pinch of hen egg lysozyme and one Roche Complete EDTA-free protease inhibitor tablet, lysed using a French press and clarified by centrifugation as described. For coencapsulation experiments, $0.2 \mathrm{~L}$ equivalents of the $\mathrm{HT}_{\mathrm{SC}}$ expressing lysate was mixed with a total of $0.2 \mathrm{~L}$ equivalent $\mathrm{sTcfp} / \mathrm{sT} y f p$ lysates at varying ratios where one part corresponds to $20 \mathrm{~mL}$ equivalents lysate. For encapsulation of a single species (control reactions and loading into $\mathrm{HT}_{\mathrm{ST}}$ shells), $0.2 \mathrm{~L}$ equiv. of shell lysate was mixed with $0.2 \mathrm{~L}$ equiv. cargo. Mixtures were incubated at room temperature overnight and then incubated with clarified $\mathrm{P}_{\text {SII }}$ lysate and purified via $1 \mathrm{~mL}$ StrepTrap columns as described and concentrated with $0.5 \mathrm{~mL} 100 \mathrm{kDa}$ MWCO spin filters to obtain sufficient fluorescent signal. For fluorescence comparisons to encapsulated ${ }_{\mathrm{ST}} \mathrm{cfp} / \mathrm{ST}_{\mathrm{T}} \mathrm{yp}$, the flow-through during sample application to the StrepTrap columns was collected.

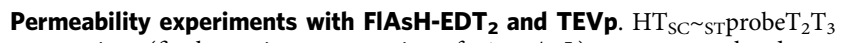
preparations (final protein concentration of $\sim 1 \mathrm{mg} / \mathrm{mL}$ ) or unencapsulated sT probe $(\sim 100 \mu \mathrm{g} / \mathrm{mL})$ in $50 \mathrm{mM}$ Tris- $\mathrm{HCl}, \mathrm{pH} 7.4,150 \mathrm{mM} \mathrm{NaCl}, 5 \mathrm{mM}$ EDTA, $5 \mathrm{mM}$ TCEP, $1 \mathrm{mM} \beta$-mercaptoethanol were incubated in the absence or presence of excess $\mathrm{P}_{\mathrm{SII}}$ in microtiter plates for sixty minutes. A FlAsH-EDT 2 and 2,3-dimercapto-1-propanol solution ( $1 \mathrm{mM}$ and $9 \mathrm{mM}$, respectively, in DMSO) was added such that the final FlAsH-EDT 2 concentration was $10 \mu \mathrm{M}$ and incubated at room temperature for $2 \mathrm{~h}$. TEV protease was then added to a final concentration of $40 \mu \mathrm{g} / \mathrm{mL}(\sim 1: 20 \mathrm{w} / \mathrm{w})$ and incubated for $2 \mathrm{~h}$.

Refer to Supplementary Methods for additional notes on the following topics: chemicals and reagents; SDS-PAGE and western blot analysis of protein preparations; fluorescence intensity, spectra readings, and fluorescence normalization; electron microscopy. Plasmid construction is summarized in Supplementary Table 1. Full-frame TEM micrographs were provided in Supplementary Fig. 10 .

Data availability. Data supporting the findings of this study are available from the corresponding author upon reasonable request.

Received: 22 February 2018 Accepted: 15 June 2018

Published online: 23 July 2018

\section{References}

1. Axen, S. D., Erbilgin, O. \& Kerfeld, C. A. A taxonomy of bacterial microcompartment loci constructed by a novel scoring method. PLoS Comput. Biol. 10, e1003898 (2014).

2. Sutter, M., Greber, B., Aussignargues, C. \& Kerfeld, C. A. Assembly principles and structure of a 6.5-MDa bacterial microcompartment shell. Science 356, 1293-1297 (2017).

3. Tanaka, S. et al. Atomic-level models of the bacterial carboxysome shell. Science 319, 1083-1086 (2008).

4. Kerfeld, C. A. et al. Protein structures forming the shell of primitive bacterial organelles. Science 309, 936-938 (2005).

5. Klein, M. G. et al. Identification and structural analysis of a novel carboxysome shell protein with implications for metabolite transport. J. Mol. Biol. 392, 319-333 (2009).

6. Cai, F. et al. The pentameric vertex proteins are necessary for the icosahedral carboxysome shell to function as a $\mathrm{CO}_{2}$ leakage barrier. PLoS ONE 4, e7521 (2009).

7. Aussignargues, C., Paasch, B. C., Gonzalez-Esquer, R., Erbilgin, O. \& Kerfeld, C. A. Bacterial microcompartment assembly: the key role of encapsulation peptides. Commun. Integr. Biol. 8, e1039755 (2015).

8. Fan, C. et al. Short N-terminal sequences package proteins into bacterial microcompartments. Proc. Natl Acad. Sci. USA 107, 7509-7514 (2010).

9. Kinney, J. N., Salmeen, A., Cai, F. \& Kerfeld, C. A. Elucidating essential role of conserved carboxysomal protein $\mathrm{CcmN}$ reveals common feature of bacterial microcompartment assembly. J. Biol. Chem. 287, 17729-17736 (2012).

10. Cameron, J. C., Wilson, S. C., Bernstein, S. L. \& Kerfeld, C. A. Biogenesis of a bacterial organelle: the carboxysome assembly pathway. Cell 155, 1131-1140 (2013).

11. Abdul-Rahman, F., Petit, E. \& Blanchard, J. L. The distribution of polyhedral bacterial microcompartments suggests frequent horizontal transfer and operon reassembly. J. Phylogen. Evolution. Biol. 1118 (2013).

12. Lawrence, J. G. \& Roth, J. R. Evolution of coenzyme $B_{12}$ synthesis among enteric bacteria: evidence for loss and reacquisition of a multigene complex. Genetics 142, 11-24 (1996). 
13. Parsons, J. B. et al. Biochemical and structural insights into bacterial organelle form and biogenesis. J. Biol. Chem. 283, 14366-14375 (2008).

14. Conrado, R. J., Mansell, T. J., Varner, J. D. \& DeLisa, M. P. Stochastic reaction-diffusion simulation of enzyme compartmentalization reveals improved catalytic efficiency for a synthetic metabolic pathway. Metab. Eng. 9, 355-363 (2007).

15. Jakobson, C. M., Tullman-Ercek, D., Slininger, M. F., \& Mangan, N. M. A systems-level model reveals that 1,2-Propanediol utilization microcompartments enhance pathway flux through intermediate sequestration. PLoS Comput. Biol. 13, e1005525 (2017).

16. Sampson, E. M. \& Bobik, T. A. Microcompartments for B12-dependent 1,2-propanediol degradation provide protection from DNA and cellular damage by a reactive metabolic intermediate. J. Bacteriol. 190, 2966-2971 (2008).

17. Kim, E. Y. \& Tullman-Ercek, D. Engineering nanoscale protein compartments for synthetic organelles. Curr. Opin. Biotechnol. 24, 627-632 (2013).

18. Plegaria, J. S. \& Kerfeld, C. A. Engineering nanoreactors using bacterial microcompartment architectures. Curr. Opin. Biotechnol. 51, 1-7 (2018).

19. Cai, F., Bernstein, S. L., Wilson, S. C. \& Kerfeld, C. A. Production and characterization of synthetic carboxysome shells with incorporated luminal proteins. Plant Physiol. 170, 1868-1877 (2016).

20. Lassila, J. K., Bernstein, S. L., Kinney, J. N., Axen, S. D. \& Kerfeld, C. A. Assembly of robust bacterial microcompartment shells using building blocks from an organelle of unknown function. J. Mol. Biol. 426, 2217-2228 (2014).

21. Choudhary, S., Quin, M. B., Sanders, M. A., Johnson, E. T. \& SchmidtDannert, C. Engineered protein nano-compartments for targeted enzyme localization. PLoS ONE 7, e33342 (2012).

22. Jakobson, C. M., Slininger Lee, M. F. \& Tullman-Ercek, D. De novo design of signal sequences to localize cargo to the 1,2-propanediol utilization microcompartment. Protein Sci. 26, 1086-1092 (2017).

23. Erbilgin, O., Sutter, M. \& Kerfeld, C. A. The structural basis of coenzyme a recycling in a bacterial organelle. PLoS Biol. 14, e1002399 (2016).

24. Schmidt, T. G. M., Koepke, J., Frank, R. \& Skerra, A. Molecular interaction between the Strep-tag affinity peptide and its cognate target, streptavidin. J. Mol. Biol. 255, 753-766 (1996).

25. Price, G. D. \& Badger, M. R. Isolation and characterization of high $\mathrm{CO}(2)-$ requiring-mutants of the Cyanobacterium Synechococcus PCC7942: two phenotypes that accumulate inorganic carbon but are apparently unable to generate CO(2) within the Carboxysome. Plant Physiol. 91, 514-525 (1989).

26. Zakeri, B. et al. Peptide tag forming a rapid covalent bond to a protein, through engineering a bacterial adhesin. Proc. Natl Acad. Sci. USA 109, E690-E697 (2012).

27. Goedhart, J. et al. Structure-guided evolution of cyan fluorescent proteins towards a quantum yield of 93\%. Nat. Commun. 3, 751 (2012).

28. Kremers, G. J., Goedhart, J., van Munster, E. B. \& Gadella, T. W. Jr. Cyan and yellow super fluorescent proteins with improved brightness, protein folding, and FRET Forster radius. Biochemistry 45, 6570-6580 (2006).

29. Arosio, P., Elia, L. \& Poli, M. Ferritin, cellular iron storage and regulation. IUBMB Life 69, 414-422 (2017).

30. Kerfeld, C. A., Aussignargues, C., Zarzycki, J., Cai, F. \& Sutter, M. Bacterial microcompartments. Nat. Rev. Microbiol. 16, 277-290 (2018).

31. Dougherty, W. G., Cary, S. M. \& Parks, T. D. Molecular genetic analysis of a plant virus polyprotein cleavage site: a model. Virology 171, 356-364 (1989).

32. Griffin, B. A., Adams, S. R. \& Tsien, R. Y. Specific covalent labeling of recombinant protein molecules inside live cells. Science 281, 269-272 (1998).

33. Cai, F. et al. The structure of $\mathrm{CcmP}$, a tandem bacterial microcompartment domain protein from the beta-carboxysome, forms a subcompartment within a microcompartment. J. Biol. Chem. 288, 16055-16063 (2013).

34. Larsson, A. M., Hasse, D., Valegard, K. \& Andersson, I. Crystal structures of beta-carboxysome shell protein $\mathrm{CcmP}$ : ligand binding correlates with the closed or open central pore. J. Exp. Bot. 68, 3857-3867 (2017).
35. Sargent, F. et al. A synthetic system for expression of components of a bacterial microcompartment. Microbiology 159, 2427-2436 (2013).

36. Seebeck, F. P., Woycechowsky, K. J., Zhuang, W., Rabe, J. P. \& Hilvert, D. A simple tagging system for protein encapsulation. J. Am. Chem. Soc. 128 4516-4517 (2006)

37. Giessen, T. W. \& Silver, P. A. A catalytic nanoreactor based on in vivo encapsulation of multiple enzymes in an engineered protein nanocompartment. Chembiochem 17, 1931-1935 (2016).

38. Cai, F., Sutter, M., Bernstein, S. L., Kinney, J. N. \& Kerfeld, C. A. Engineering bacterial microcompartment shells: chimeric shell proteins and chimeric carboxysome shells. ACS Synth. Biol. 4, 444-453 (2015).

39. Menon, B. B., Heinhorst, S., Shively, J. M. \& Cannon, G. C. The carboxysome shell is permeable to protons. J. Bacteriol. 192, 5881-5886 (2010).

40. Slininger Lee, M. F., Jakobson, C. M. \& Tullman-Ercek, D. Evidence for improved encapsulated pathway behavior in a bacterial microcompartment through shell protein engineering. ACS Synthetic Biol. 68, 3857-3867 (2017).

\section{Acknowledgements}

We thank Behzad Rad and Francesca Manea for plasmids and fruitful discussion of SpyTag/SpyCatcher technology, the labs of Adam Deutschbauer and Krishna Niyogi for instrument use and Denna Kwang for assistance with cloning. This work was supported by the National Institutes of Health, National Institute of Allergy and Infectious Diseases (NIAID) grant 1R01AI114975-01 with infrastructure support from the US Department of Energy, Basic Energy Sciences, Contract DE-FG02-91ER20021.

\section{Author contributions}

A.H. designed and performed the experiments and wrote the paper. M.S. initiated the project and assisted with experimental design. N.S. generated reagents, assisted with experiments and performed electron microscopy. C.A.K. supervised the project, contributed to experimental design and co-wrote the paper. All authors discussed the results and contributed critical feedback during the preparation of the manuscript.

\section{Additional information}

Supplementary Information accompanies this paper at https://doi.org/10.1038/s41467018-05162-z.

Competing interests: The authors declare no competing interests.

Reprints and permission information is available online at http://npg.nature.com/ reprintsandpermissions/

Publisher's note: Springer Nature remains neutral with regard to jurisdictional claims in published maps and institutional affiliations.

Open Access This article is licensed under a Creative Commons Attribution 4.0 International License, which permits use, sharing, adaptation, distribution and reproduction in any medium or format, as long as you give appropriate credit to the original author(s) and the source, provide a link to the Creative Commons license, and indicate if changes were made. The images or other third party material in this article are included in the article's Creative Commons license, unless indicated otherwise in a credit line to the material. If material is not included in the article's Creative Commons license and your intended use is not permitted by statutory regulation or exceeds the permitted use, you will need to obtain permission directly from the copyright holder. To view a copy of this license, visit http://creativecommons.org/ licenses/by/4.0/

(C) The Author(s) 2018 